\title{
X-ray computed tomography coupled to Digital Volume Correlation applied to a stationary crack case
}

\author{
Y. Barranger ${ }^{1, \mathrm{a}}$, P. Doumalin ${ }^{1}$, J.C. Dupré ${ }^{1}$, A. Germaneau ${ }^{1}$, S. Hédan ${ }^{2}$, and V. Valle ${ }^{1}$ \\ ${ }^{1}$ Institut Pprime, UPR 3346, CNRS - Université de Poitiers - ENSMA, 86962 Futuroscope \\ Chasseneuil Cedex, France \\ ${ }^{2}$ Université de Poitiers, Laboratoire HydrASA, UMR 6269, Ecole Supérieure d'Ingénieurs de \\ Poitiers, 86022 Poitiers Cedex, France
}

\begin{abstract}
The aim of this study is to evaluate 3D numerical simulation and 2D theory on a single edge notch cracked specimen loaded in mode I in light of experimental data. The three displacement components through the whole specimen were measured by Digital Volume Correlation coupled to X-ray micro-computed tomography. The theoretical approach gives higher results than the experimental ones, which means that the plane-stress theory is not relevant of the reality in this particular case. On the contrary, the numerical simulation made on the finite element software CASTEM gives results similar to the experimental ones with differences equivalent to the accuracy of the experimental method.
\end{abstract}

\section{Introduction}

On rupture of materials in the case of stationary crack, several experimental methods have been conducted around the crack tip to measure the out-of-plane displacement components (like interferometric techniques [1,2]) and the two in-plane displacements (with 2D measurements methods $[3,4]$ ). However, displacement components were accessible only at the free surface of the solid with these methods, which can not insure that the 2D theory is still validated at the core of the solid. In order to acquire displacement components at the bulk of the solid, it is then necessary to perform measurements through the whole solid.

Numerical methods can provide full field data through the whole solid thanks to the validation of displacement measurements performed at the surface. However, to validate these numerical models at each point of the mesh, it is necessary to perform three-dimensional measurements at the bulk of the solid to insure that results obtained numerically through the whole solid correspond to the reality.

To perform bulk measurement of the three displacement components, Digital Volume Correlation (DVC) has been employed. This technique is coupled with tomographic methods given volume data like X-ray computed tomography $(\mathrm{X} \mu \mathrm{CT})$ [5-7], or optical slicing tomography [7-9], or either confocal microscopy [10].

In this study, X $\mu \mathrm{CT}$ is coupled with DVC and applied on a SEN (Single Edge Notch) cracked specimen loaded in mode I. Firstly, experimental displacement fields are compared to theoretical

\footnotetext{
a e-mail : yoann.barranger@univ-poitiers.fr
} 
ones. Finally, these results are compared to the one obtained by a numerical finite element simulation.

\section{Theoretical expressions}

In planar elasticity, two different theoretical approaches give expressions for strain and displacement fields, they are called plane-stress and plane-strain approaches. Westergaard [11] proposed an expression for the displacement fields which characterises the in-plane components of displacements at the vicinity of the crack tip. Based on this expression, Williams [12] developed these formulations by mean of a Taylor expansion.

In this study, the first two terms of the Williams expansion have been chosen to calculate the theoretical displacements with a focus on the plane-stress approach case:

$$
\left\{\begin{array}{l}
u_{x}(r, \theta)=\frac{K_{I}}{\mu} \sqrt{\frac{r}{2 \pi}} \cos \frac{\theta}{2}\left(\frac{1-v}{1+v}+\sin ^{2} \frac{\theta}{2}\right)-\frac{\sigma}{E} r \cos \theta+0 \times r^{n / 2} \\
u_{y}(r, \theta)=\frac{K_{I}}{\mu} \sqrt{\frac{r}{2 \pi}} \sin \frac{\theta}{2}\left(\frac{2}{1+v}-\cos ^{2} \frac{\theta}{2}\right)+\frac{v \sigma}{E} r \sin \theta+0 \times r^{n / 2} \\
u_{z}(r, \theta, z)=-\frac{v}{E} \int_{0}^{z}\left(K_{I} \sqrt{\frac{2}{r \pi}} \cos \frac{\theta}{2}-\sigma\right) d z
\end{array}\right.
$$

$\mu$ is the second coefficient of Lamé (also called elastic shear modulus) and is expressed by $\mu=E /(2(1+v)) . E$ is the Young modulus and $v$ is the Poisson's ratio of the material. The cylindrical coordinate $(r, \theta, z)$ is centred on the crack tip $(x, y, z)=(0,0,0)$, with the plane $z=0$ located at the semithickness of the material. The stress intensity factor $K_{I}$ is based on Tada's formulation [13].

\section{Numerical simulation}

The numerical model is based on an existing model initially developed to compare out-of-plane displacements obtained numerically with the one obtained by interferometry [14]. This extension to a 3D case with a finite plate has been developed in order to validate boundary conditions and the behaviour law from surface measurement data [15]. It was developed under the finite element software CASTEM.

In this study, the specimen is under a uniaxial load perpendicular to the cracked surface (crack opening mode I). Because of this loading condition and of the geometry, the problem is symmetric according to the cracked and ligament plane $(y=0)$ and to the medium plane $(z=0)$. Consequently, only one quarter of the specimen is considered in the numerical modelling for this mode I case (figure 1). The crack front is imposed straight and is perpendicular to the free surface of the specimen. Around the crack front, a circular mesh is applied with a radius $(r)$ equal to the crack length $(a)$. Because of the high displacement gradients near the crack tip at the free surface, the mesh is refined in this area: the element size of this circular mesh increases with the radius on the surface, and is gradually reduced below the free surface. As shown on figure 1, the rest of the model is added to the half of cylinder already modelled in order to fully simulate the quarter of the specimen.

An elastic linear law has been applied for this model As a boundary condition, a zerodisplacement is imposed at the point $(0,0,0)$ located at the crack front for the $x$ direction. The other boundary conditions used in the numerical simulation are linked to the previously described symmetries:

$$
\begin{cases}u_{y}(M)=0, & M(x, y, z) \in P_{l i g} \\ u_{z}(M)=0, & M(x, y, z) \in P_{\text {med }}\end{cases}
$$




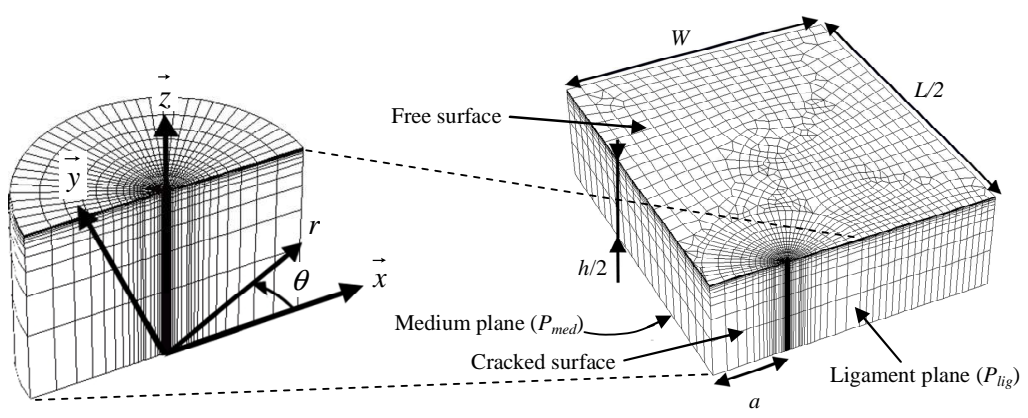

Fig. 1. Mesh of a quarter of a specimen around the circular mesh

\section{Experimental methods and material}

\subsection{Digital Volume Correlation}

DVC is the extension to 3D cases of the Digital Image Correlation (DIC) [7-9]. As for DIC, DVC technique enables the measurements of displacement fields between two images of a specimen, each one representing a different mechanical state (a reference state and a deformed state). For DVC, images are volumes containing a 3D grey level distribution. A 3D virtual grid is defined in the reference image, and at each point of this grid a subset $D$ is defined by grey levels of the neighbourhood voxels (extension of pixel in 3D). The intercorrelation of the subset between both states gives an estimation of the displacement of each point of the virtual grid.

We consider $\underline{X}$ and $\underline{x}$ the coordinates in voxels of the same point for the reference state and the deformed state. These coordinates are linked by the material transformation $\phi$, with $\underline{x}=\phi(\underline{X})$. For a subset $D$ centred on the point $\underline{X}_{0}$ at the reference state, $\phi$ is approximated by its expansion at the first order corresponding to a rigid body motion combined with a homogenous gradient:

$$
\phi(\underline{X})=\underline{X}+u(\underline{X}) \approx \underline{X}+u\left(\underline{X}_{0}\right)+\frac{\partial u}{\partial \underline{X}}\left(\underline{X}_{0}\right) \cdot\left(\underline{X}-\underline{X}_{0}\right)
$$

The displacement $u\left(\underline{X}_{0}\right)$ of the subset centre defines the parameters of the rigid body translation $(u, v, w)$. The local displacement gradient includes the rigid body rotation and the local deformation of the subset. The local displacement gradient is characterised by its nine parameters:

$\left(\frac{\partial u}{\partial x}, \frac{\partial u}{\partial y}, \frac{\partial u}{\partial z}, \frac{\partial v}{\partial x}, \frac{\partial v}{\partial y}, \frac{\partial v}{\partial z}, \frac{\partial w}{\partial x}, \frac{\partial w}{\partial y}, \frac{\partial w}{\partial z}\right)$

To determine the position, a first-gradient minimization is used in the DVC process. By means of a trilinear interpolation, the calculation of grey level variations between two adjacent voxels is performed on the deformed image. With this method, it is then possible to achieve a subvoxel precision similar to the one obtained with the DIC [7].

\subsection{X-ray micro-computed tomography}

To acquire the volume images necessary to perform the DVC technique, a laboratory tomography device was used, allowing us to have a minimal resolution about $10 \mu \mathrm{m}$. It is composed of an X-ray source which transmits X-rays through the specimen disposed on a rotation plate. A radiography is then recorded for each angular position with a CCD camera. From these different images, the 3D image is reconstructed by means of a back-projection algorithm [16]. Local differences of density inside the specimen are depicted on images by the grey level distribution. It is used by DVC in order to follow the local material kinematics. 
Several materials can give natural contrast to X-rays because of their heterogeneities. However, to use DVC technique, the contrast has to be sufficient and not all materials present a sufficient contrast to suit this technique. Therefore, the inclusion of small dense markers in the material during the cast of the specimen can be necessary in order to have a sufficient contrast for DVC [7].

\subsection{Materials and geometrical specifications}

For this study, two different materials were necessary: one for the specimen and one for the enclosed particles. To choose the material of the specimen, a compromise was made between three criterions. Firstly, the specimen was casted from liquid resins in order to make easier the inclusion and the blending of the particles. Secondly, the material was chosen with a low Young's modulus in order to have displacements along the three directions higher than DVC accuracy. Finally, in order to remain in elastic assumptions used for the numerical simulation, the material had to have a quasi-pure elastic behaviour until large strain. For this study, polyurethane was chosen in order to fit these criterions.

Copper particles were chosen and included in the polyurethane at $10 \%$ of the total mass of the specimen. As an assumption, mechanical gradients involved by particles are assumed to remain localized to the particle scale, which induces that the specimen behaviour is assumed to be homogenous according to our measurement scale.

Mark tracking technique [17] was performed to obtain the mechanical characteristics $(E, v)$ of the material on a normalized tensile test specimen casted at the same time as the cracked specimen.

The geometrical dimensions of the SEN specimen (figure 1) are: length $L=70 \mathrm{~mm}$, width $W=29 \mathrm{~mm}$, and thickness $h=21 \mathrm{~mm}$. These dimensions were chosen to fit the experimental tensile apparatus placed in the laboratory tomography device and to have a sufficient thickness in order to have a better view of the 3D effects. With these dimensions, the DVC subset is $31 \times 31 \times 31$ voxels $^{3}$ with a voxel size of $45 \times 45 \times 45 \mu \mathrm{m}^{3}$. According to [7], with these parameters the displacement measurement uncertainty is around 0.05 voxel. The crack length of this SEN specimen is $a=7.5 \mathrm{~mm}$ and was created with a cutter blade. Finally, a $36 \mathrm{~N}$ load was applied on the specimen by the experimental tensile apparatus.

\section{Results and analysis}

In figure 2 , the displacement results $\left(u_{x}, u_{y}, u_{z}\right)$ are plotted for the plane stress, the numerical and the experimental approaches at $36 \mathrm{~N}$. As the theoretical formulations for $u_{x}$ and $u_{y}$ do not depend on $z$, they are plotted on a plane. For the two other approaches and $u_{z}$ for the theory, results are presented for the whole specimen, showing displacement fields at the free surface $(z=h / 2)$ and a bit on the thickness. Full results at specific slices are shown in [18].

For $u_{x}$, some differences can be noted between the theory and the two other approaches, whereas numerical and experimental results seem to be similar. Concerning $u_{y}$, results seem to be equivalent between all approaches. Finally for $u_{z}$, significant differences appear between the theory and the two other approaches.

To quantify these differences, figure 3 is showing directly these differences between firstly the theory and the experiment, and secondly between the numerical simulation and the experiment. For the differences between theoretical and experimental displacements, results are plotted for only half of the specimen so as to have a better view of the evolution of the differences along the thickness. For the differences between numerical and experimental approaches, as there is no significant evolution along the thickness, only the representation on the full specimen is shown. 


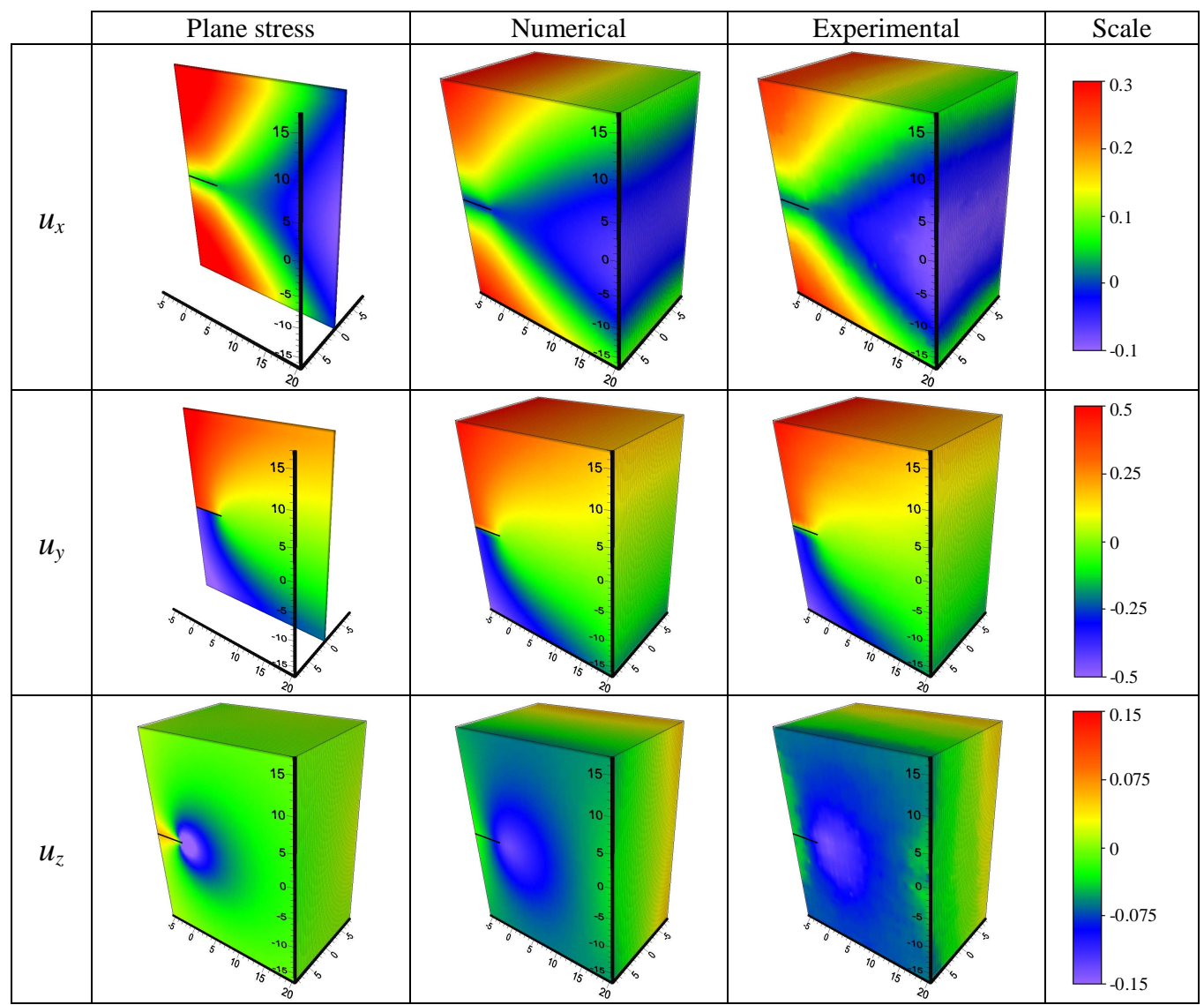

Fig. 2. $u_{x}, u_{y}$ and $u_{z}$ for theoretical, numerical and experimental approaches.

From the first part of figure 3, it can be seen that main differences are located far from the crack tip for $u_{x}$ and at the back of it. Hence, Williams formulation for this component is not relevant of the real behaviour on a cracked finite thick specimen.

For $u_{y}$, differences are also located at the back of the crack, but they do not have significant amplitude. Consequently, it can be assumed that this component is less sensitive to the assumptions made for the theory like for the boundary conditions. Indeed, Williams formulation is based on the assumption of infinite plate, whereas the experiment is a finite model.

Finally for $u_{z}$, differences are located at the vicinity of the crack tip. This area corresponds to the location where 3D effects are usually observed with $2 \mathrm{D}$ measurements at the free surface. With this conical evolution of the differences along the thickness, maximums of amplitude are reached at the free surfaces.

The second part of figure 3 shows that differences between the numerical simulation and the experiment are really low for each component. Sinusoidal evolutions of differences can be seen (for $u_{x}$ the sinusoidal evolution has a radial distribution around the crack front, whereas for $u_{y}$ and $u_{z}$ the evolutions are respectively along $y$ and $z$ directions). The amplitude of these differences is about $0.02 \mathrm{~mm}$, which corresponds to the systematic error of the DVC observed in previous studies [7]. 


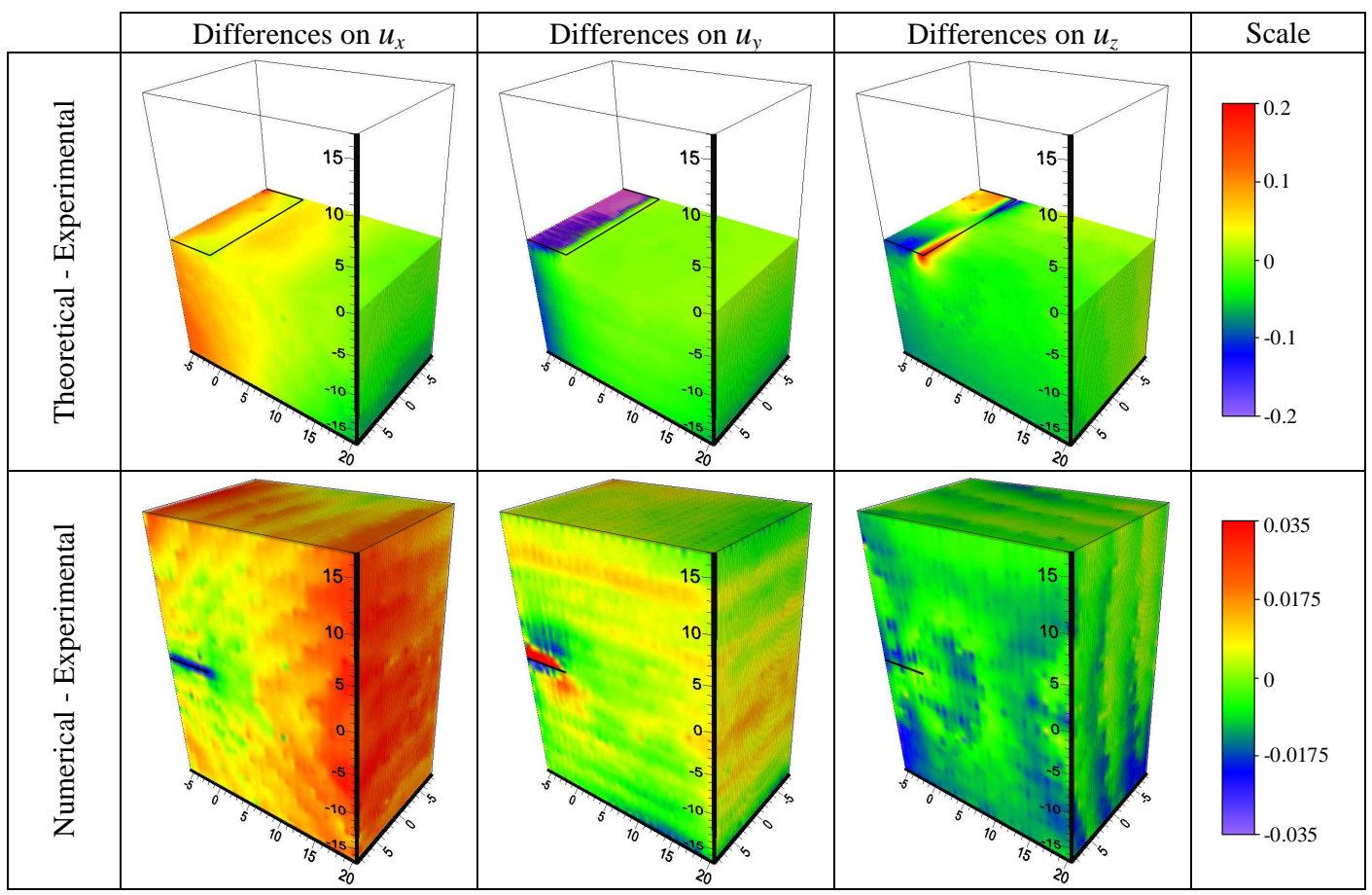

Fig. 3. Differences between theoretical and experimental results, and between numerical and experimental results for the three components

Small differences can be seen for $u_{y}$ near the crack front. A first assumption was made to explain these differences, saying that the behaviour law included in the numerical simulation for the material does not take into account a possible non-linearity at high stress. Hence, a non-linear law was included in the numerical simulation from the data obtained previously by mark tracking technique. Figure 4 shows the behaviour law of our material and the differences between the numerical simulation made for the linear law and the non-linear one.

(a)

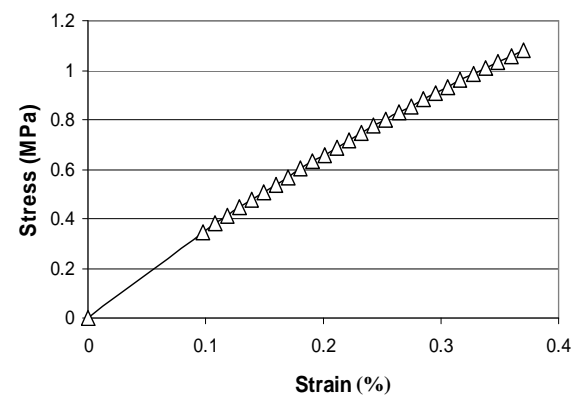

(b)

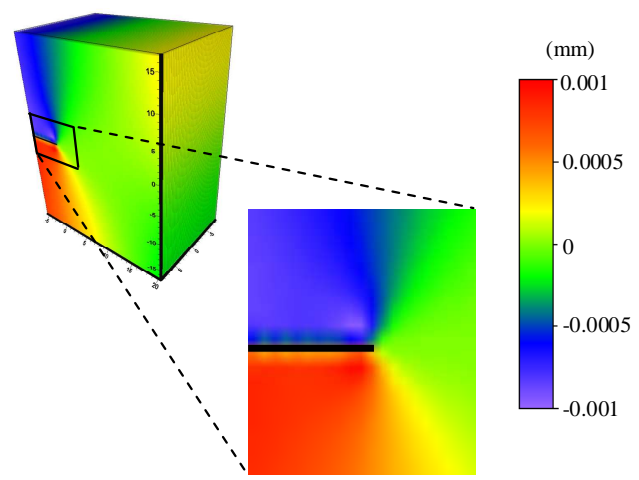

Fig. 4. Non-linear behaviour law input in the numerical simulation (a) and difference between the linear and the non-linear law for $u_{y}$ (b) 
From this figure, it can be seen that the amplitude of differences between the non-linear and the linear law is not enough significant to explain the local phenomenon at the crack front for $u_{y}$. Indeed, differences observed on figure 3 between numerical (with the linear law) and experimental results for $u_{y}$ at the vicinity of the crack tip have an amplitude of $0.015 \mathrm{~mm}$, whereas differences on figure 4.b between the linear and the non-linear laws show a maximum of amplitude about $0.001 \mathrm{~mm}$ on the same zone. Other assumptions were made about the boundary conditions and a possible crack opening during the test but are still under investigation.

\section{Conclusion}

To perform bulk displacement measurements, DVC coupled with X-ray micro-computed tomography appeared to be a powerful method.

The comparison between the plane-stress theory, the numerical simulation and the experimental results allowed the evaluation of the limits of each one of these methods for the estimation of displacement fields in a cracked specimen loaded in mode I.

The 2D theory is revealed to be not relevant of the reality because of large differences with the experimental results. Only the component $u_{y}$ shows small differences between the plane-stress approach and the experiment, whereas $u_{x}$ shows large differences far from the crack as well as $u_{z}$ next to the crack tip. These two components are then more sensitive to the assumptions made for the establishment of the theoretical formulations than $u_{y}$.

However, the 3D numerical simulation made on the finite element software CASTEM seems to be relevant of the reality. Differences between the two approaches are small, their amplitude are equivalent to the accuracy of DVC technique.

\section{References}

1. R.D. Pfaff, P.D. Washabaugh, W.G. Knauss, International Journal of Solids and Structures 32(6/7), 939-955 (1995)

2. L. Humbert, V. Valle, M. Cottron, International Journal of Solids and Structures 38, 5493-5504 (2000)

3. M.A. Sutton, J.L. Turner, Y.J. Chao, H.A. Bruck, T.L. Chae, International Journal of Fracture 53, 201-228 (1992)

4. J. Arakawa, K. Takahashi, International Journal of Fracture 86, 289-300 (1997)

5. B.K. Bay, T.S. Smith, D.P. Fyhrie, M. Saad, Experimental Mechanics 39, 217-226 (1999)

6. L. Liu, E.F. Morgan, Journal of Biomechanics 40, 3516-3520 (2007)

7. A. Germaneau, P. Doumalin, J.C. Dupré, NDT\&E International 41, 407-415 (2008)

8. A. Germaneau, P. Doumalin, J.C. Dupré, Experimental Mechanics 47, 523-532 (2007)

9. A. Germaneau, P. Doumalin, J.C. Dupré, Strain 43, 207-218 (2007)

10. C. Franck, S. Hong, S.A. Masharinee, D.A. Tirrell, G. Ravichandran, Experimental Mechanics 47, 427-438 (2007)

11. H.M. Westergaard, Journal of Applied Mechanics 6, 49-53 (1939)

12. M.L. Williams, Journal of Applied Mechanics 24, 109-114 (1957)

13. H. Tada, P.C. Paris, G.R. Irwin, The Stress Analysis of Cracks Handbook (Paris Productions, 1985)

14. L.Humbert, V. Valle M. Cottron, ZAMM Z Angew. Math. Mech. 80 (2000)

15. S. Hédan, V. Valle, M. Cottron, Experimental Mechanics 50, 401-412 (2010)

16. L.A. Feldkamp, L.C. Davis, J.W. Kress, Journal of Optical Society of America 1(6), 612-619 (1984)

17. N. Bretagne, V. Valle, J.C. Dupré, NDT\&E International 39(4), 290-298 (2005)

18. Y. Barranger, P. Doumalin, J.C. Dupré, A. Germaneau, S. Hédan, V. Valle, Engineering Fracture Mechanics 76, 2371-2383 (2009) 